\title{
Thermal diffusivity measurement of ceramic materials used in spraying of TBC systems
}

\author{
The influence of materials' morphology and (re)manufacturing processes
}

\author{
Grzegorz Moskal $^{1} \cdot$ Marta Mikuśkiewicz $^{1}$ (D) $\cdot$ Anna Jasik $^{1}$ \\ Received: 16 October 2018 / Accepted: 19 July 2019/Published online: 7 August 2019 \\ (C) The Author(s) 2019
}

\begin{abstract}
The basic goal of this article was thermal diffusivity characterization of ceramic materials used in thermal barrier coating (TBC) systems for depositions of the insulation layer and characterization of the materials' morphology and remanufacturing process. The base material was oxide $8 \mathrm{YSZ}\left(\mathrm{ZrO}_{2} \times 8 \mathrm{Y}_{2} \mathrm{O}_{3}\right)$, which is usually dedicated to deposition of an insulating top layer in TBC systems. The data related to thermal properties such as thermal diffusivity and thermal conductivity are widely presented in the literature, but there is lack of information about the morphological form of investigated materials, and the presented results vary widely. Data on thermal properties based on the literature sources are inadequate for the real morphological form of materials used in the experiment (e.g., massive or single crystalline material vs. plasma-sprayed coatings), which consequently gives an unsatisfactory accuracy of the obtained numerical simulations by MES methods. This article presents the characterization of thermal diffusivity of the commercial 8 YSZ ceramic material synthesized or remanufactured by different routes, which is investigated in the forms of pressed powder pellet (two commercial nano-sized powders with different morphologies), sintered pellets (one commercial powder, solid-state co-precipitated reacted powder of 8YSZ type), and a two-layered coating system of In625 + NiCrAlY/8YSZ type. The range of analysis included morphological investigations of different types of powders in initial conditions and after remanufacturing (sintering, thermal spraying) as well as the thermal diffusivity analysis by the laser flash method. The obtained data were corrected by porosity factor and compared to each other. The best similarity for obtained thermal diffusivity data was found for commercial powers of $\mathrm{HOSP}^{\mathrm{TM}}$ type after pressing and sintering processes and calculated (2layered model) value of thermal diffusivity for two-layered system of In625/8YSZ TBS system. The results showed that there are significant differences in thermal diffusivity values for materials with different morphological forms.
\end{abstract}

Keywords TBC $\cdot$ LFA $\cdot$ Thermal diffusivity $\cdot 8 Y$ SZ $\cdot$ Insulation properties $\cdot$ Morphology

\section{Introduction}

The basic role of ceramic top-coats in thermal barrier coatings systems is thermal insulation of the metallic substrate during operations at high temperature with additional assistance of a strong aggressive environment. The typical temperature of the analyzed ceramic layer at its

Marta Mikuśkiewicz

marta.mikuskiewicz@polsl.pl

1 Institute of Materials Engineering, Silesian University of Technology, Krasińskiego 8 Str., 40-019 Katowice, Poland surface is up to $1200-1400{ }^{\circ} \mathrm{C}$. The temperature of $\mathrm{Ni}(\mathrm{Co}) \mathrm{CrAlY}$ or $\mathrm{NiAl}(\mathrm{Pt})$ bond-coats, localized below the top-coat, should not be higher than $900{ }^{\circ} \mathrm{C}[1,2]$. The function of the top-coat is to decrease the temperature to at least $300{ }^{\circ} \mathrm{C}$, under the assumption that the thickness of the ceramic insulation is generally no higher than $300 \mu \mathrm{m}$. This assumption requires the knowledge of the thermal properties of the ceramic materials used in TBC deposition, especially the thermal conductivity or diffusivity in a wide temperature range [3-5]. Thermal conductivity and diffusivity are important in the numerical modeling of the temperature and the stress distribution in the ceramic layer. 
However, the major problem is related to the precision of the obtained thermal data, as well as the morphological accordance of the tested ceramic materials with that of the finally obtained materials during the deposition processes of ceramic coatings using different plasma methods. Different processing routes of the same feedstock material allow us to produce materials having the same chemical and phase composition, but totally different morphologies and, in consequence, thermal properties [6-14].

The main goal of this article is the overall characterization of the $\mathrm{ZrO}_{2} \times 8$ mass $\% \quad \mathrm{Y}_{2} \mathrm{O}_{3}$ ceramic material, which is typically used as a feedstock powder for deposition of the insulation layer in TBC systems and characterization of thermal diffusivity of these materials according to their morphological forms created with different routes of synthesis or remanufacturing.

\section{Experimental}

The first step of investigations was related to the characterization of morphology and thermal diffusivity measurement of commercial powders of 8YSZ type obtained by three different methods: plasma remelting process (commercially available powder of $\mathrm{HOSP}^{\mathrm{TM}}$ type was used), spray drying, and crushing and milling. The range of investigations in this part included phase analysis of powders (Jeol JDX-7S diffractometer), their chemical composition (ICP-OES-inductively coupled plasma-optical emission spectrometer-Ultima 2 ICP OES spectrometer-basic elements and additives, HFIR-highfrequency infrared-Coulomat 702 by Strohlein-carbon and sulfur, THE_high-temperature extraction-ON-mat 8500 by Strohlein - oxygen and nitrogen) as well as their morphology (Hitachi 3400-N scanning electron microscopy), powder size by laser diffraction method, (Mastersizer-Hydro 2000S Malvern Inc.) and thermal diffusivity measured by laser flash method (LFA 427 by Netzsch).

Specimens dedicated to laser flash analysis were pressed (25 $\mathrm{MPa}$ ) in a mechanical press to form cylindrical pellets with a thickness of $3 \mathrm{~mm}$ and diameter $10 \mathrm{~mm}$. The test was carried out in temperature range $25-1500{ }^{\circ} \mathrm{C}$ with steps of $250{ }^{\circ} \mathrm{C}$. The obtained data were corrected due to porosity factor in accordance with Eq. (1):

$\frac{\alpha}{\alpha_{0}}=1-\frac{4}{3} \rho$

where $\rho$-porosity and $\alpha_{0}$-actual thermal diffusivity. In all cases, the porosity of the pressed pellets was ca. $40 \%$. The porosity measurement was made by Archimedes method and was verified by images analysis.

The thermal diffusivity measurement was made on the pressed and pressed/sintered specimens with known thickness, as well as on the TBC system built from three layers: substrate material, bond-coat and insulating ceramic layer. In analyzed case only data for ceramic layer were showed, as a result of calculation based on Proteus software (Netzsch) and 2 layered model with heat loss. As the first layer, the data obtained for the substrate material with bond-coat (IN625/NiCrAlY) were used. As the second layer, the data for only the ceramic sublayer were calculated on the basis of thermal diffusivity of the IN625/ NiCrAlY first layer and data obtained for all TBC systems (IN625/NiCrAlY/8YSZ).

The second part of analysis was related to the characterization of $8 \mathrm{YSZ}$ materials after different routes of synthesis. The pellet sintered at $1500{ }^{\circ} \mathrm{C} / 24 \mathrm{~h}$ and made from plasma remelting of $8 \mathrm{YSZ}$ powder was used as a model specimen. The next specimen was synthesized by solidstate reaction (SSR) of $\mathrm{ZrO}_{2}$ and $\mathrm{Y}_{2} \mathrm{O}_{3}$ feedstock powders with an adequate ratio of $8 \mathrm{YSZ}$ formula. The input materials in SSR methods are oxide powders, which are mixed in appropriate proportions, homogenized and finally sintered. In the SSR route, powders of $\mathrm{ZrO}_{2}$ and $\mathrm{Y}_{2} \mathrm{O}_{3}$ were weighed stoichiometrically and mixed. The mixtures were homogenized by wet milling in ethanol for $15 \mathrm{~min}$, then dried and finally sintered in a "Degussa" vacuum press at $1300{ }^{\circ} \mathrm{C}$ for $2 \mathrm{~h}$ with an additional $15 \mathrm{MPa}$ load pressure. These methods are relatively simple, but the structure of the final product is often inhomogeneous. In the chemical methods (e.g. co-precipitation, sol-gel), which was used to prepare the last specimen (co-precipitation-C-P), the 8YSZ powder is obtained from liquid solutions. Precursors are dissolved and mixed at the molecular level, which enhances homogeneity. However, the reagents are often more expensive; therefore, these methods are preferred when there is high homogeneity but a large amount of material are needed [5-8]. The starting materials were zirconium oxychloride $(\mathrm{ZrOCl} 2.8 \mathrm{H} 2 \mathrm{O}, 99.99 \%)$ and yttrium nitrate $\left(\mathrm{Y}\left(\mathrm{NO}_{3}\right)_{3} \cdot 6 \mathrm{H}_{2} \mathrm{O}\right)$. They were weighed to obtain the equimolar cation ratio, dissolved in distilled water and magnetically stirred for several hours. Citric acid (CA) was added to form metal-citrate complex compounds at c.a. $80{ }^{\circ} \mathrm{C}$, and finally, ethylene glycol was added to initiate polycondensation after evaporation of solvents. The powder, called organic precursor, was calcined at $1000{ }^{\circ} \mathrm{C}$ in the muffle furnace and then vacuum-sintered into pellets in the same condition as SSR powders $\left(1300^{\circ} \mathrm{C} / 2 \mathrm{~h} /\right.$ $15 \mathrm{MPa}$ ). Both SSR and C-P synthesized samples were additionally sintered at similar conditions as plasmaremelted specimens.

The last part of the experiment consisted of characterization of plasma-sprayed ceramic coatings deposited from the spray-dried commercial powder of 8YSZ type. TBC coating was fabricated by atmospheric plasma spraying. Before spraying, the superalloy IN625 substrates were 
Fig. 1 Grain size distribution of the analyzed powders

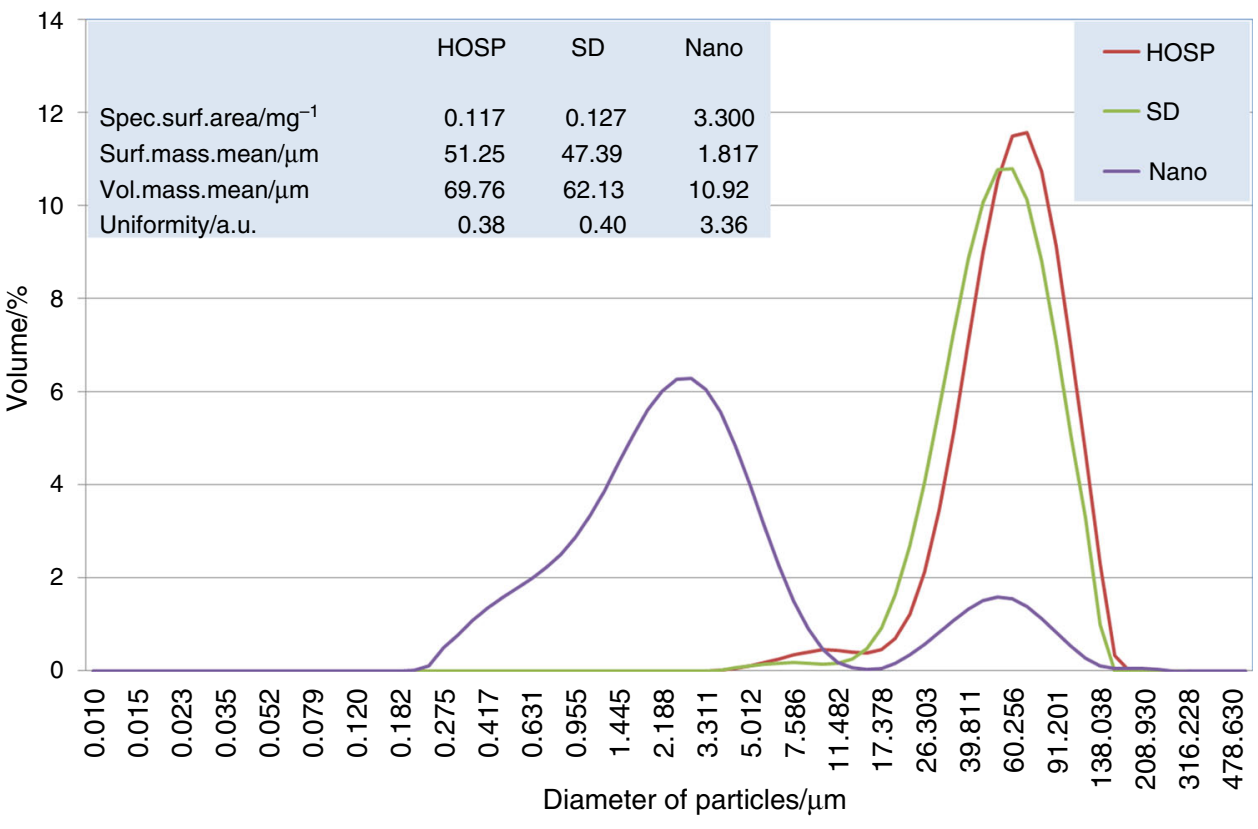

immersed in ethanol for ultrasonic cleaning. Next, the substrate was degreased and grit-blasted with corundum in order to increase the bonding strength. Feedstock powder was thermally sprayed using an $\mathrm{Ar} / \mathrm{H}_{2}$ APS torch (F4 type). Thermal spray parameters were as follows: current 570A, voltage $55 \mathrm{~V}$, flow rate of primary gas $100 \mathrm{SCFH}$ $\left(\mathrm{SCFH}+0.472 \mathrm{Lmin}^{-1}\right)$, feedstock given rate $7 \mathrm{~g} / \mathrm{min}$, spray distance $90 \mathrm{~mm}$, spray angle $90^{\circ}$ and spray velocity $35 \mathrm{~mm} / \mathrm{s}$. Additionally, the microstructure of the obtained coating was shortly described with special attention to porosity.

\section{Results and discussion}

Results of granule size distribution obtained during laser diffraction analysis are shown in Fig. 1. Commercial powders of 8YSZ type obtained by the plasma method and spray drying process had a very similar unimodal distribution of granule diameter. In the case of nano-sized 8YSZ material, the bimodal structure was detected with dominated small granules and diameter in the range of ca. $200 \mathrm{~nm}$ to $12 \mu \mathrm{m}$. This result presents the differences in the powder morphology and shape, which are important parameters from the thermal conductivity point of view.

Analysis of chemical composition of powders revealed that there is no significant difference in chemical constituents of 8YSZ powders obtained by plasma remelting, spray drying or crushing and milling to nano-size. Special attention was given to different types of impurities present in the powders, because this factor very strongly influences the thermal diffusion of ceramic materials. But in analyzed cases, the level of impurities in all powders was very similar (Table 1). A small difference was observed in the case of phase constituents of 8 YSZ powders obtained by different methods: the monoclinic phase fraction was the

Table 1 Chemical composition of the analyzed powders

\begin{tabular}{llll}
\hline Chemical composition/mass/\% & HOSP $^{\mathrm{TM}} 8 \mathrm{YSZ}$ & SD 8YSZ & "Nano" 8YSZ \\
\hline $\mathrm{Gd}$ & - & - & - \\
$\mathrm{Zr}$ & $\mathrm{Rest}$ & Rest & Rest \\
$\mathrm{Y}$ & $6.10 \pm 0.10$ & $6.20 \pm 0.10$ & $6.20 \pm 0.10$ \\
$\mathrm{Al}$ & $0.17 \pm 0.008$ & $0.21 \pm 0.008$ & $0.21 \pm 0.008$ \\
$\mathrm{Si}$ & $<0.10$ & $<0.20$ & $<0.20$ \\
$\mathrm{Cu}$ & $<0.01$ & $<0.01$ & $<0.01$ \\
$\mathrm{Ti}$ & $<0.067 \pm 0.003$ & $0.020 \pm 0.003$ & $0.020 \pm 0.003$ \\
$\mathrm{~S}$ & $0.004 \pm 0.0004$ & $0.002 \pm 0.0002$ & $0.002 \pm 0.0002$ \\
$\mathrm{C}$ & $0.018 \pm 0.002$ & $0.033 \pm 0.002$ & $0.033 \pm 0.002$ \\
$\mathrm{O}_{2}$ & $12.9 \pm 0.08$ & $12.8 \pm 0.08$ & $12.8 \pm 0.08$ \\
$\mathrm{~N}_{2}$ & $101 \mathrm{ppm} \pm 10$ & $132 \mathrm{ppm} \pm 10$ & $134 \mathrm{ppm} \pm 10$ \\
\hline
\end{tabular}




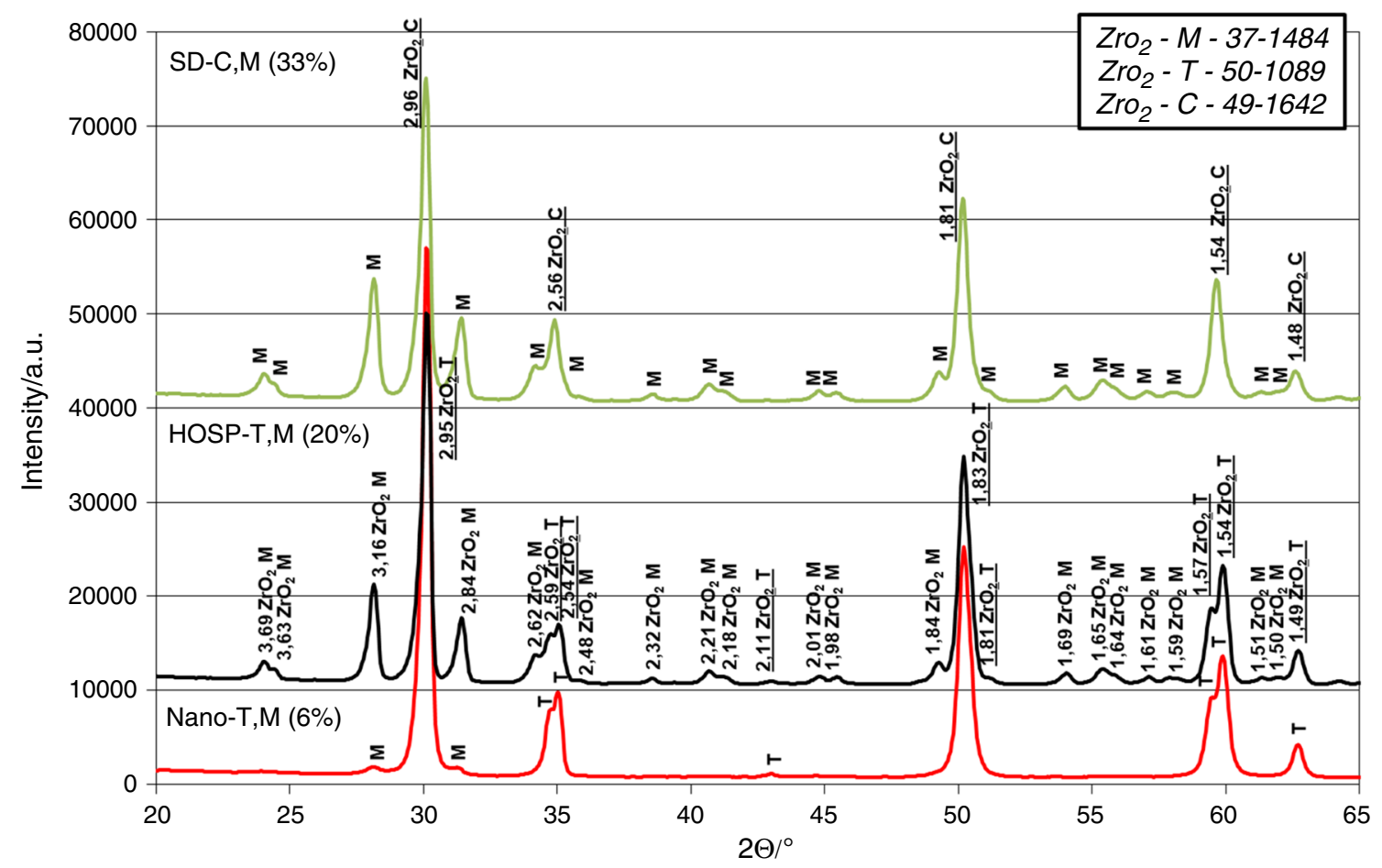

Fig. 2 Phase composition of the analyzed 8YSZ powders

highest in the case of spray-dried powder and the smallest was detected for nano-sized powder (Fig. 2). The structural aspects of $\mathrm{ZrO}_{2}$ oxide, especially its lattice form, is an important factor influencing the phonon scattering effects. In Fig. 2, only structural identification of zirconia was related to the presence of cubic, tetragonal and monoclinic forms of $\mathrm{ZrO}_{2}$.

Much more varied is the morphology of 8YSZ type granules, where the most important factor which influences the internal structure of powders is the technology of manufacturing. Generally, all three powders have a typical morphology characteristic of the method of manufacturing. First of them, which is obtained by the plasma method, is smooth on the granule surfaces and empty inside (hollow sphere). The next powder obtained by spray drayed process is characterized by complex internal structure consisted small particles of basic ceramic materials and voids, formed to the shape of granules which are much easier susceptible to plasma spraying process. The last type of powder (nano-sized) has morphology typical for the crushing and milling process (Fig. 3).

The first step of investigation of thermal properties consists of laser flash analysis of pressed pellets from plasma remelting, spray drying and nano-sized 8YSZ powders (Fig. 4). The obtained data suggest that the nanosized powder had the lowest thermal conductivity independently before or after correction with Eq. 1. These data are in accordance with the literature data where nano-sized
8YSZ ceramic is characterized by better thermal insulation properties than micro-sized ceramic of the same type $[9,15]$. When compared to plasma remelting and spraydried 8YSZ powders, a slightly lower thermal diffusivity was found for plasma-treated powder probably due to a little higher internal porosity than its spray-dried version.

The plasma-treated 8YSZ powder was chosen for further investigations. In this part of investigations, the mechanically pressed pellets were sintered at temperature $1500{ }^{\circ} \mathrm{C}$ during $24 \mathrm{~h}$. Additionally, for comparison, 8YSZ ceramic was synthesized by the solid-state reaction and coprecipitation method and analyzed by laser flash method as well. Comparison of thermal diffusivity data (Fig. 5) obtained for pressed only plasma-treated powder and the same pellet after the sintering process at temperature $1500{ }^{\circ} \mathrm{C}$ showed that the thermal diffusivity increased significantly, which is related to the lower porosity level (ca. 15\%-image analysis results).

The solid-state sintering and co-precipitation method gives us the possibility to obtain ceramic materials with a much lower porosity level than those obtained by sintering of commercial $8 \mathrm{YSZ}$ powders. In the analyzed cases, it was 10 and lower than 5\%, respectively. Laser flash analysis (Fig. 6) of these powders showed that the highest thermal diffusivity level was noted for pellets obtained from powders synthesized by the co-participation method.

Observed differences are related mainly to the lowest level of porosity in the case of C-P manufactured powders. 
Fig. 3 Morphology of the analyzed 8YSZ powders

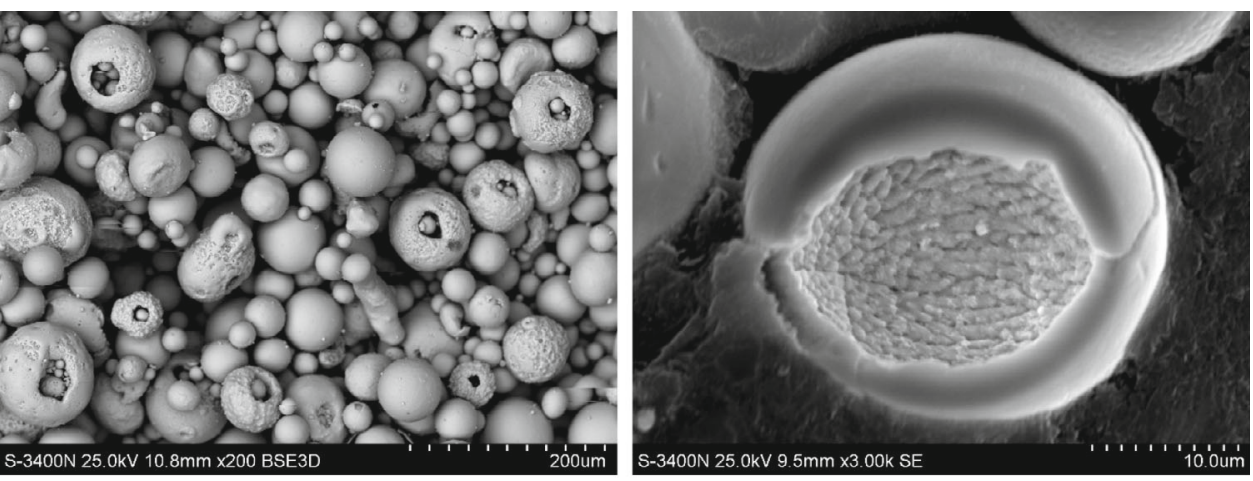

plasma remelting

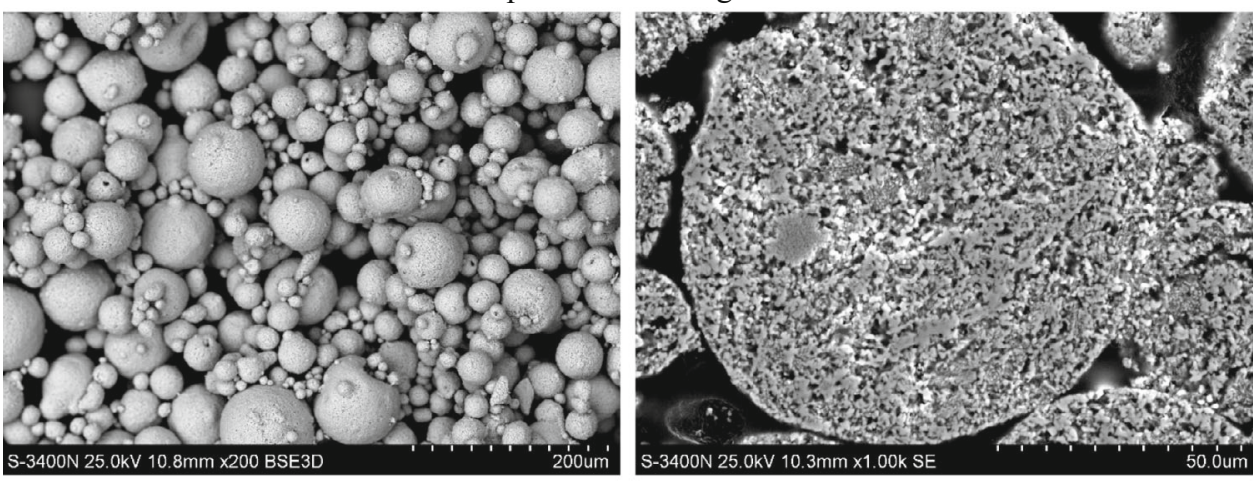

spray dried

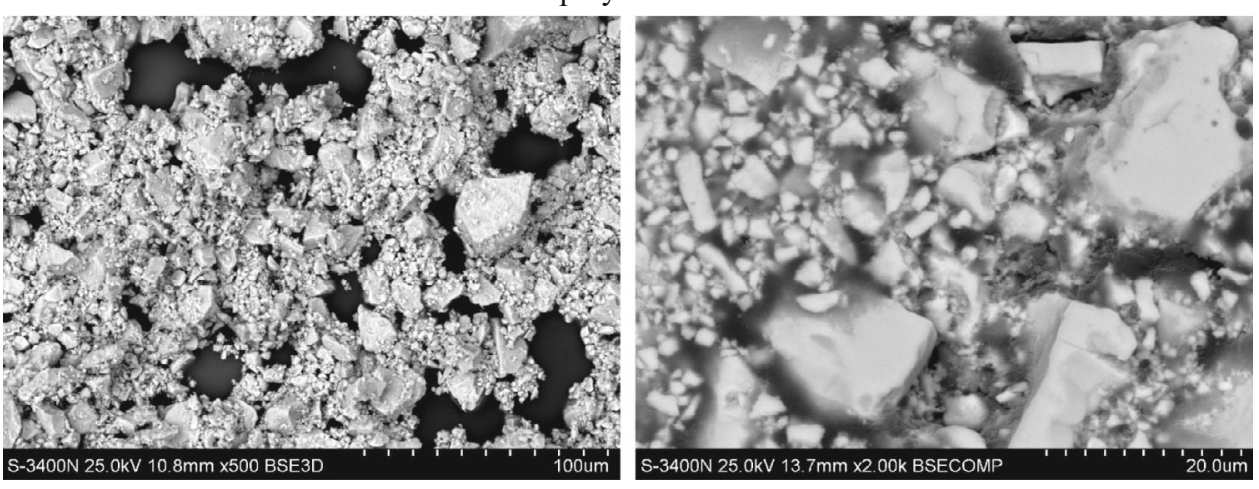

Nano
The differences in phase composition do not have strong influence on insulation properties of the obtained materials. The results of phase constituents of the investigated powders analyzed by the XRD method are shown in Fig. 7, where the basic difference is the presence of the monoclinic phase in the sintered commercial powder.

The last stage of investigations is the comparison of thermal diffusivity of the insulating ceramic layer deposited by atmospheric plasma spraying in the form of thermal barrier coating with data obtained for 8YSZ materials synthesized by different routes or remanufactured from commercial powders. Morphology of the typical TBC system with 8YSZ type of ceramic top-coat is shown in Fig. 8. The upper part of the figure shows the light microscopy view of the overall TBC system with the presentation of the ceramic top layer and NiCrAlY bondcoat deposited on the IN625 substrate alloy. Details of the ceramic microstructure with pores and cracks obtained by the scanning electron microscopy method are shown on the left side of the figure. The voids' architecture (pores and cracks) is the most important structural factor that influences the insulation properties of TBC systems. The next parameter is the chemical and phase constituents of the material used as an outer layer in TBC systems. In this case the data showed phase constituent of analyzed layer were showed on the right side in the form of diffraction data and information about presence of tetragonal, cubic and monoclinic zirconia.

The thickness of the analyzed ceramic layer was ca. $200 \mu \mathrm{m}$, and its porosity was 5\%. The XRD analysis of 


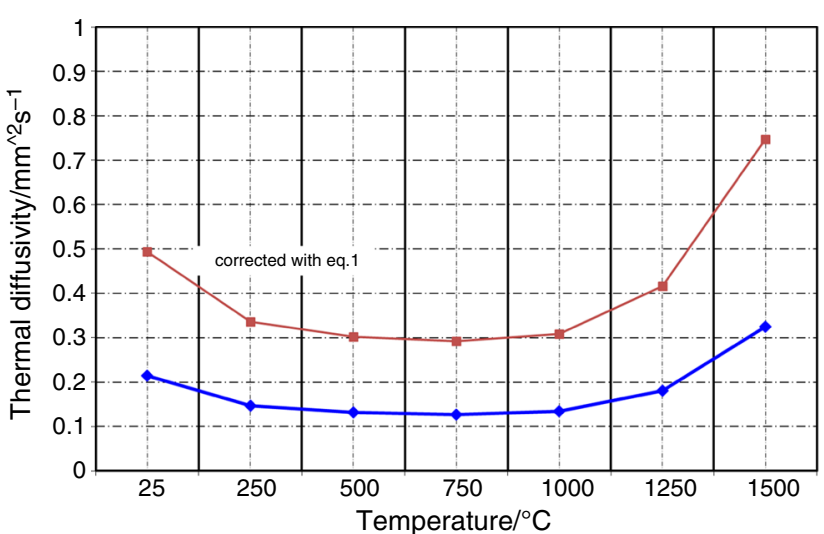

HOSPTM8YSZ

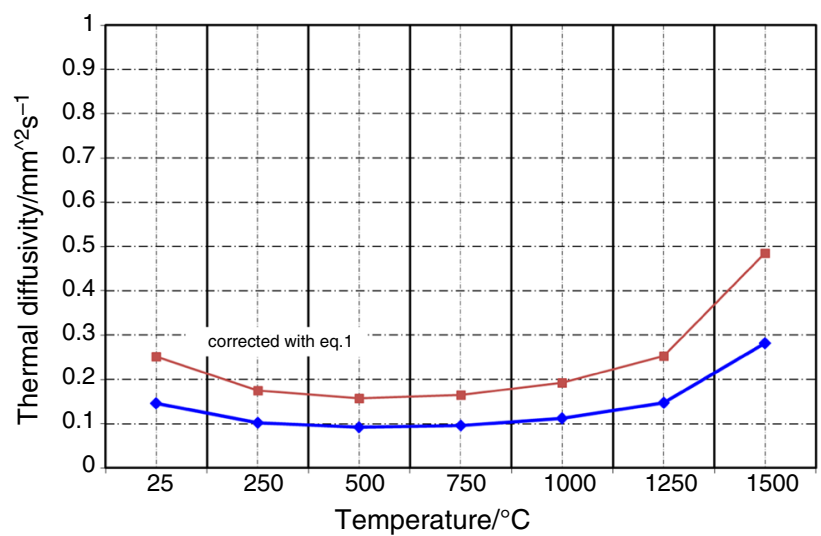

Nano 8YSZ

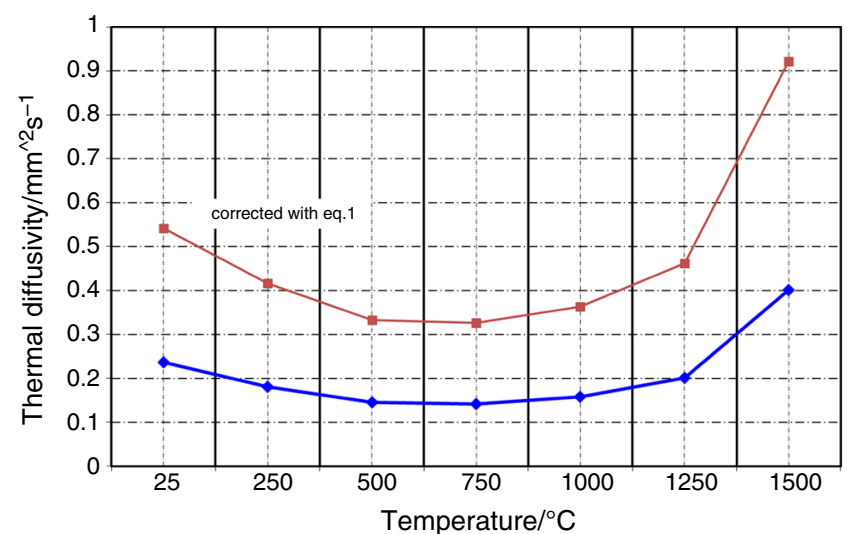

SD 8YSZ

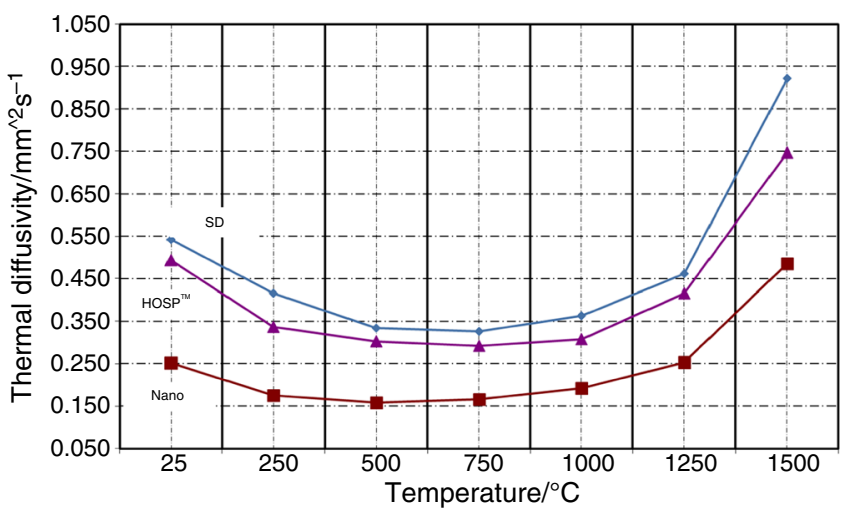

Summary plot of corrected curves

Fig. 4 Thermal diffusivity of 8YSZ powders with and without correction in accordance with equation no. 1

Fig. 5 Thermal diffusivity of plasma-remelted 8YSZ powder after mechanical pressing and after sintering process

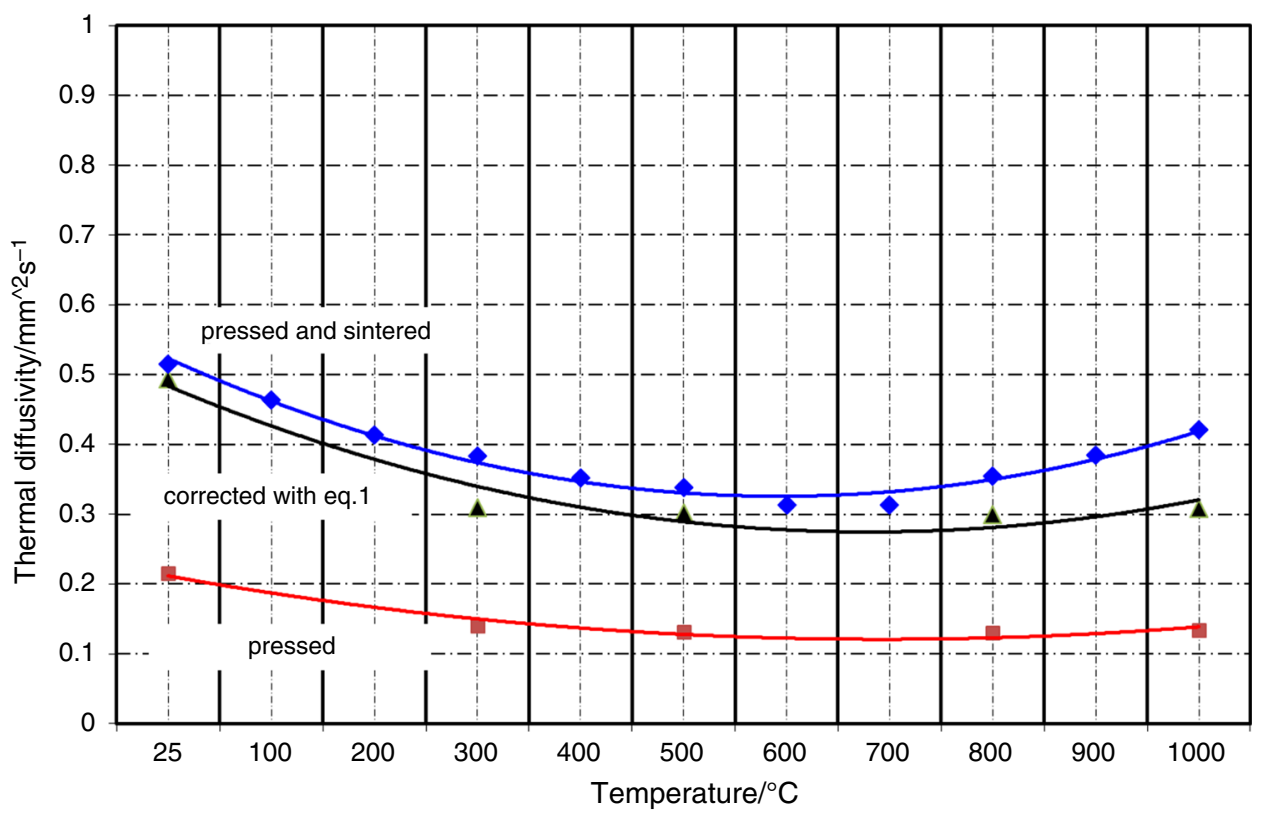


Fig. 6 Thermal diffusivity of plasma-remelted $8 Y S Z$ powder after different routes of synthesis and compaction

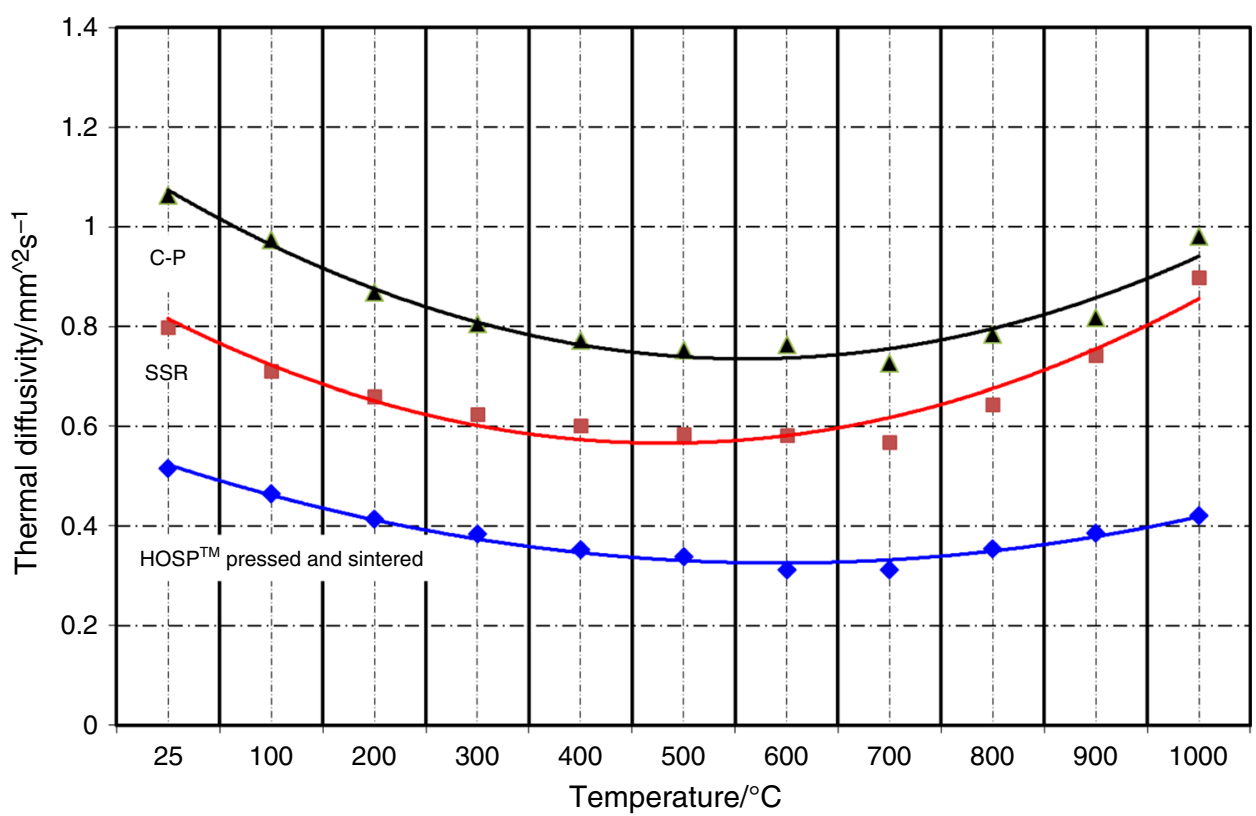

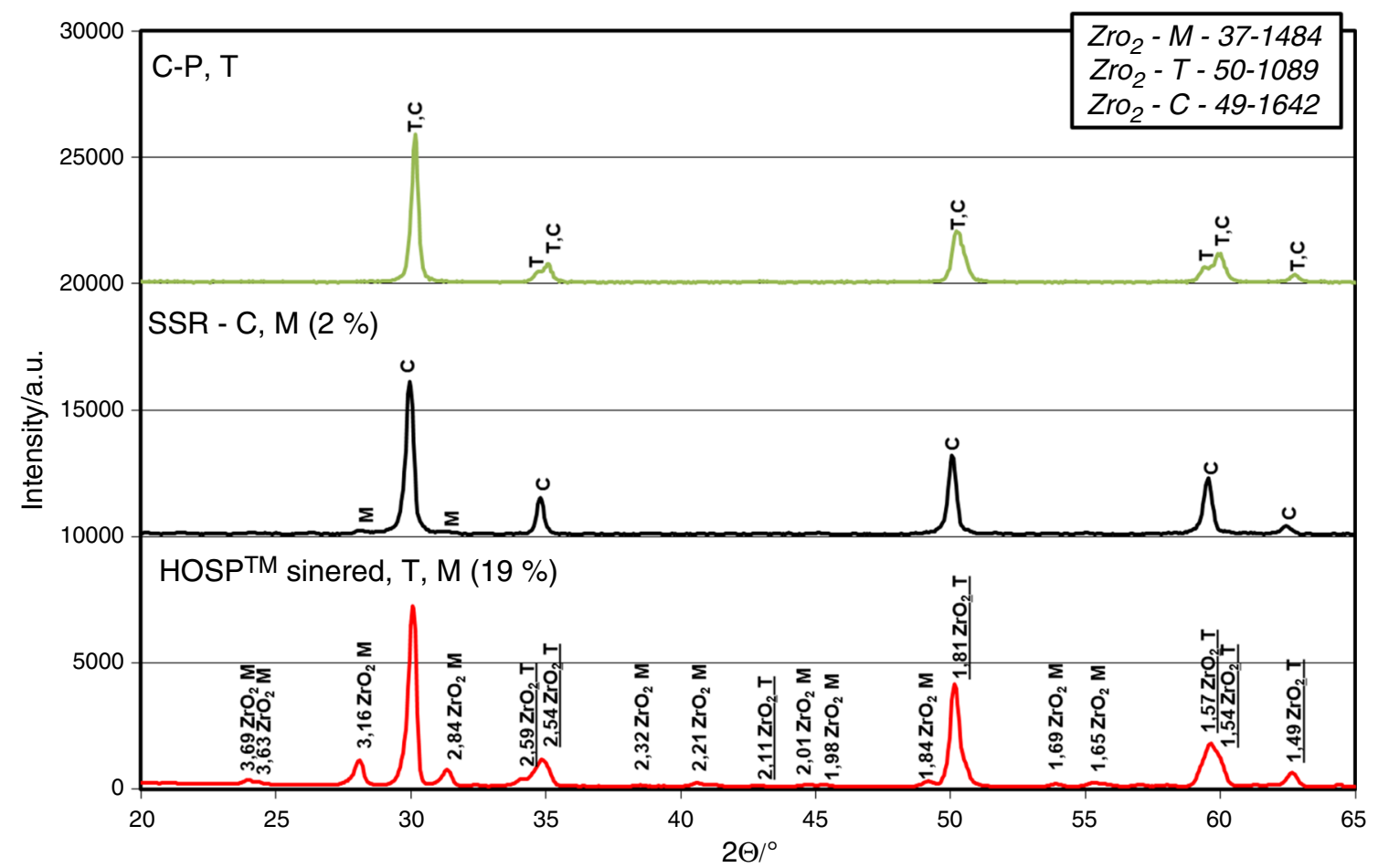

Fig. 7 Phase composition of the synthesized 8YSZ powders

phase compositions revealed that the main phase element was tetragonal and cubic zirconia with a small amount of a monoclinic compound (ca. 3\%). Firstly, the thermal diffusivity of the substrate alloy with bond-coat was determined in the next step, and the whole TBC system was investigated. On the basis of these results, the Proteus software by Netzsch was used to calculate the thermal diffusivity of the ceramic top-coat. The obtained data were fitted by a 2-layer model with heat loss correction. The 625 alloy with the NiCrAlY bond-coat was treated as the first layer. The obtained data after calculation are shown in Fig. 9 where the good accordance with the thermal diffusivity of the sintered 8YSZ pellets obtained from the HOSP $^{\mathrm{TM}}$ powder is visible.

This accordance is especially good at lower temperatures. In the temperature range from 400 to $1000{ }^{\circ} \mathrm{C}$, the 


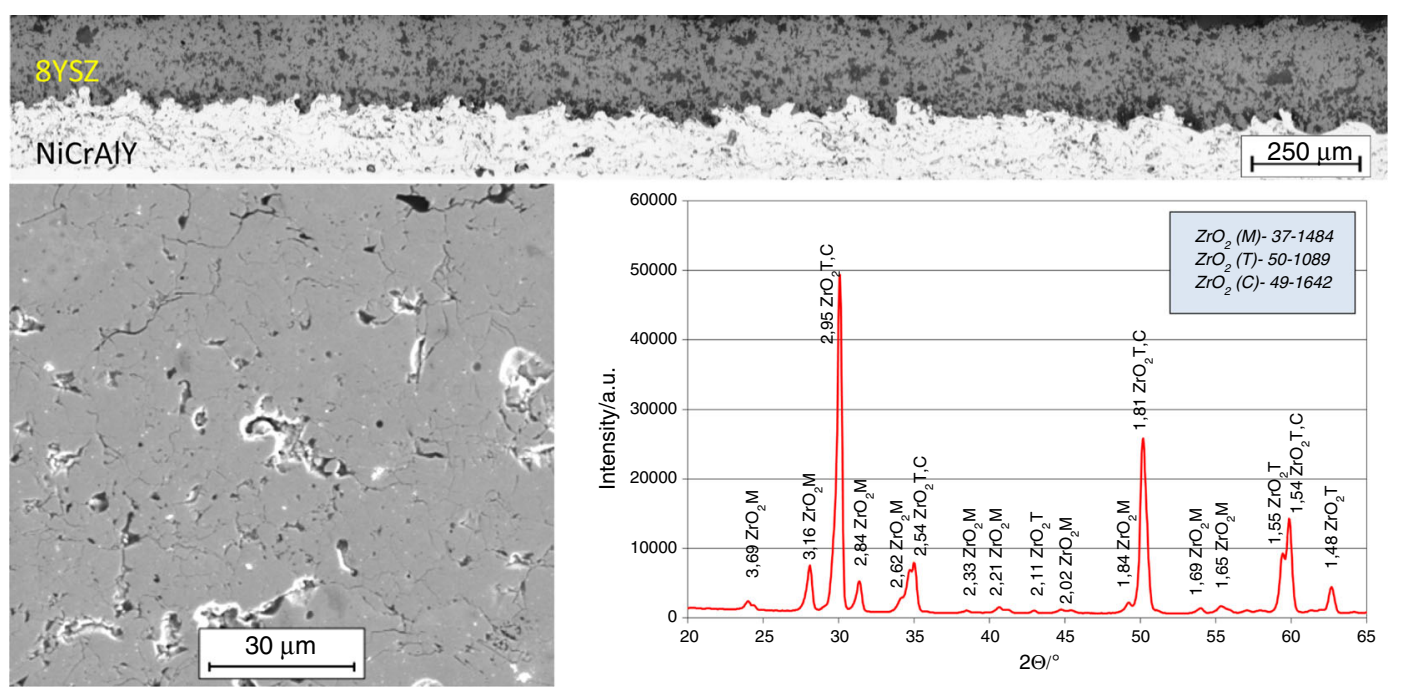

Fig. 8 Overall characteristics of the analyzed TBC system based on plasma-remelted 8YSZ feedstock powder deposited by the APS method

Fig. 9 Thermal diffusivity of plasma-treated 8YSZ powder and $8 Y S Z$ ceramic top-coat

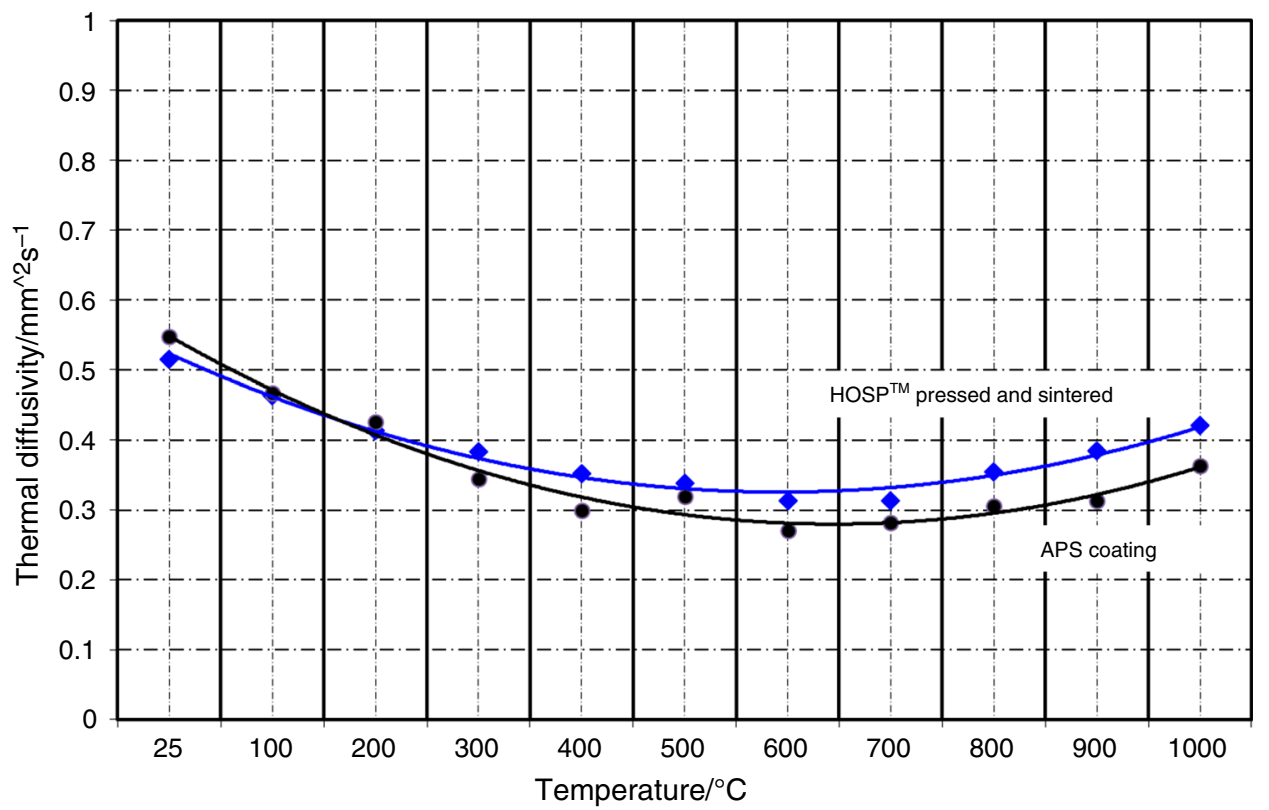

observed differences are higher due to different levels in porosity of both materials and a stronger effect of radiative transport of heat in the case of sintered specimens.

\section{Conclusions}

The present investigations revealed that the morphology of the feedstock powders is related to the methods of their synthesis, and remanufacturing routes have very strong influence on the internal structure of the final material. In consequence different thermal properties are received for the same materials from chemical and phases constituent point of view but for example remanufactured by different routes.

This information is important especially in the case of numerical simulation of temperature and stress distribution, for example in the case of thermal barrier coatings. The differences in thermal properties of $8 \mathrm{YSZ}$ can generate mistakes in developed models. The present investigations reveal that the best accordance in the thermal diffusivity value, between real component such as the 8YSZ top-coat of the TBC system and other synthesized or remanufactured 8YSZ materials, was obtained for the pellets pressed and sintered at $1500{ }^{\circ} \mathrm{C}$ from the plasma-treated feedstock powder of $8 \mathrm{YSZ}$ type. 
Acknowledgements This work was supported by the National Science center, Poland, under Grant Number 2016/21/D/ST8/01687.

Open Access This article is distributed under the terms of the Creative Commons Attribution 4.0 International License (http://creative commons.org/licenses/by/4.0/), which permits unrestricted use, distribution, and reproduction in any medium, provided you give appropriate credit to the original author(s) and the source, provide a link to the Creative Commons license, and indicate if changes were made.

\section{References}

1. Soares C. Gas turbines: a handbook of air, land and sea applications. Amsterdam: Elsevier; 2011.

2. Han J-C, Dutta S, Ekkad S. Gas turbine heat transfer and cooling technology. Boca Raton: CRC Press; 2012.

3. Hillery R, Pilsner B, McKnight R, Cook T, Hartle M. Thermal barrier coating life prediction model development. NASA Contractor Report 180807. 1988. https://ntrs.nasa.gov/archive/nasa/ casi.ntrs.nasa.gov/19890004250.pdf.

4. Miller RA. Life modelling of thermal barrier coatings for aircraft gas turbine engines. J Eng Gas Turbines Power Trans ASME. 1989;111:301-5.

5. Han M, Zhou G, Huang J, Chen S. Optimization selection of the thermal conductivity of the top ceramic layer in the double-ceramic-layer thermal barrier coatings based on the finite element analysis of thermal insulation. Surf Coat Technol. 2014;240:320-6.

6. Clarke DR, Phillpot SR. Thermal barrier coating materials. Mater Today. 2005;8:22-9.
7. Bakan E, Vaßen R. Ceramic top coats of plasma-sprayed thermal barrier coatings: materials, processes and properties. J Therm Spray Tech. 2000;1:1-2. https://doi.org/10.1007/s11666-0170597-7.

8. Bast U, Schumann E. Development of novel oxide materials for TBCs. Ceram Eng Sci Proc. 2002;23:525-32.

9. Wu J, et al. Low-thermal-conductivity rare-earth zirconates for potential thermal-barrier-coating applications. J Am Ceram Soc. 2002;85:3031-5.

10. Liu ZG, Ouyang JH, Zhou Y. Structural evolution and thermophysical properties of $(\mathrm{SmxGd} 1-\mathrm{x}) 2 \mathrm{Zr} 2 \mathrm{O} 7(0 \leq \mathrm{x} \leq 1.0)$ ceramics. J Alloys Compd. 2009;472:319-24.

11. Liu ZG, Ouyang JH, Zhou Y. Preparation and thermophysical properties of $(\mathrm{NdxGd1-x}) 2 \mathrm{Zr} 2 \mathrm{O} 7$ ceramics. J Mater Sci. 2008;43:3596-603.

12. Chen H, Gao Y, Liu Y, Luo H. Coprecipitation synthesis and thermal conductivity of La2Zr2O7. J Alloys Compd. 2009;480:843-8.

13. Zhou C, Wang N, Xu H. Comparison of thermal cycling behavior of plasma-sprayed nanostructured and traditional thermal barrier coatings. Mater Sci Eng A. 2007;452-453:569-74.

14. Yang HS, Bai GR, Thompson LJ, Eastman JA. Interfacial thermal resistance in nanocrystalline yttria-stabilized zirconia. Acta Mater. 2002;50:2309-17.

15. Patwe SJ, Ambekar BR, Tyagi AK. Synthesis, characterization and lattice thermal expansion of some compounds in the system Gd2CexZr2 - xO7. J Alloys Compd. 2005;389:243-6.

Publisher's Note Springer Nature remains neutral with regard to jurisdictional claims in published maps and institutional affiliations. 